\title{
Prismatically induced curvature and finger-tracking pressure changes in a visual capture phenomenon
}

\author{
RANDOLPH D. EASTON \\ Boston College, Chestnut Hill, Massachusetts 02167
}

\begin{abstract}
A method was developed to help elucidate the manner in which vision exerts a dominant influence over other sources of sensory information. Subjects were required to track a horizontal straight edge with their forefingers while viewing the movements of their limbs through a curve-inducing lens. Firger tracking pressure changes along the edge were transduced and monitored as a function of induced curvature orientation (convex-down, convex-up). Findings showed that greater pressure occurred when the finger was located at the low point(s) of the apparent curvature. A trend analysis indicated that the obtained pressure profiles (passive proprioception) contained large quadratic components and were exactly opposite in orientation to the shape of the visual image and subjects' reported felt curvature of the straight edge. Results were discussed in terms of central and peripheral interpretations of visual capture.
\end{abstract}

In most real-life situations, humans process visual spatial information in larger amounts and with greater acuity than any other spatial information. A likely consequence is the experimental finding that when conflicting cues regarding spatial position are encountered by a subject, the resolution is preponderantly in the direction of the visual information in spite of the true state of affairs. Empirical confirmations of the so-called dominance of vision over the other senses have been widely documented (e.g., Gibson, 1933; Nielsen, 1963; Pick, Warren, \& Hay. 1969; Rock. 1966, 1975; Ryan. 1940). It has been found, for instance, that the felt position of a hand or the heard position of a speaker viewed through laterally displacing prisms deviates little from the seen position of the target. These findings have been referred to as "visual capture," and it is assumed that vision is the cause of proprioceptive and auditory adaptation.

While the fact of dominance is established, it is generally agreed that what is more important to determine is the manner in which vision exerts its control. The present report describes a method developed to help elaborate the nature of the mechanism underlying visual dominance phenomena. Gibson (1933) deseribed an example of visual dominance involving the optical transformation of objectivity straight to subjectively curved contours. Festinger (Festinger, Burnham, Ono, \& Bamber, 1967) later affirmed this observation. If a subject wearing a curve-inducing lens tracks a straight edge with his finger, the felt excursion of the arm, hand, and finger, in tandem, reportedly corresponds to the

The author wishes to thank Ronald Shor, Al Forsyth, John Limber, and Peter Gray for their helpful comments and discussion at various stages of this research. Requests for reprints should be sent to Randolph D. Easton, Psychology Department, Boston College, Chestnut Hill, Massachusetts 02167. viewed curved movements of the limb. This occurs in spite of the fact that the limb is moving in an objectively straight path. The curved visual image, it is maintained, dominates the conscious processing of the proprioceptively straight edge.

In order to explore this particular phenomenon further, a technique was developed to transduce and monitor pressure changes of a forefinger tracking a horizontal straight edge while viewed through a curve inducing prism. It was reasoned that subjects may not, in fact. track a straight edge with constant finger pressure. To the extent that pressure changes along the edge vary as a reliable function of orientation-of-curvature viewing conditions, the effects of active or passive proprioception on experienced curvature ("visual capture") need to be assessed. While it is true that the curved visual image is correlated with experienced curvature orientation, other covariates of felt curvature need to be specified in order to understand the precise nature of visual dominance in this instance.

\section{METHOD}

\section{Apparatus and Materials}

A schematic of the apparatus is displayed in Figure 1. The tracking device and accompanying pressure transduction assembly consisted of a horizontal $50-\mathrm{cm}$-long aluminum straight edge which was affixed to the front top surface of a $60 \times 20 \mathrm{~cm}$ board placed at about the height of the subject's chest. The board was hinged along the back to a rigid vertical support and suspended by rubber strips at the front edge. Such an arrangement allowed the board to pivot up and down on its back fulcrum in a vertical-radial direction. The stiffness of the rubber suspension (precisely described below) was adjusted to prevent subjects' reported awareness of movement of the board in space but at the same time allow electromechanical monitoring of its movement.

Actual pressure transduction was accomplished by affixing a $10-\mathrm{cm}$ rod beneath the front edge at the center of the pivot board. The rod hung from a screw vertically and rested just adjacent to the pinion connector of a frictionless potentiometer. A thin slice of surgical tubing was stretched around the pinion in order to create a 


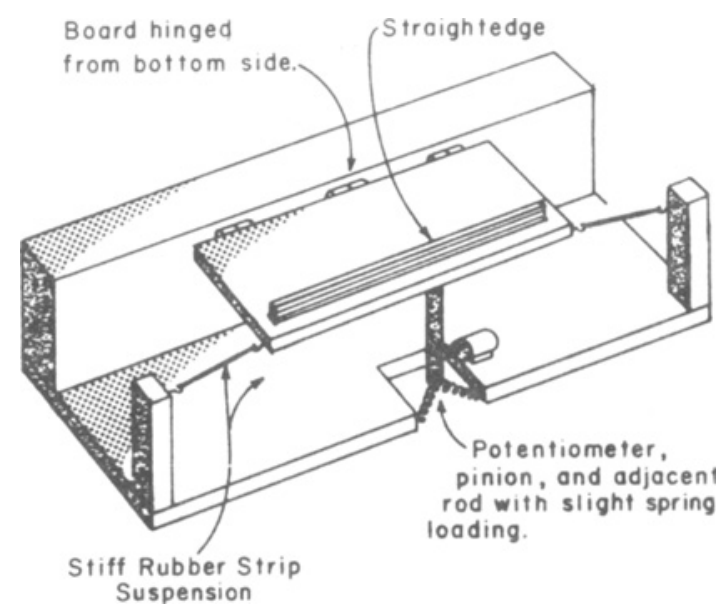

Figure 1. Schematic diagram of the hand-finger pressure transducer. The rubber supension, hinges, and potentiometer assembly were not visible to the subject.

good contact surface and avoid rod slippage. A light spring loading of the rod against the pinion insured that downward movement of the pivot board resulted in radial movement of the potentiometer pinion via downward rod movement. The voltage output of the potentiometer was amplified and displayed on an oscilloscope. The hinges. rubber suspension, potentiometer apparatus. and oscilloscope were not in view of the subject.

Calibration of the pressure transducer over the small range of pressures encountered in the experiment indicated that (1) a unit weight placed anywhere along the straight edge resulted in equal vertical-radial displacements of the board. (2) unit increases in the weight resulted in proportional unit increases in vertical-radial displacement. and (3) the same equilibrium point was regained after removing any amount of displacement. As an index of pressure needed to displace the straight edge in space, a suspension-stiffness constant was determined to be equal to $.15 \mathrm{deg}$ of radial movement per gram of weight placed on the pivot board and edge.

A $16-\mathrm{mm}$ motion picture camera was placed $4 \mathrm{ft}$ in front of the subject in such a position that the straight edge and adjacent oscilloscope display were simultaneously in view. Motion-picture photographs taken from that perspective included data on the exact lateral displacement of the subject's finger along the straight edge and a corresponding oscilloscope record of finger tracking pressure. Both lateral finger displacement and finger tracking pressure could be readily measured from enlarged negative images of each frame. Inter- and intrajudge differences were found to be random, yielding reliability coefficients of .95 and above.

The subjects viewed the straight edge monocularly (right eye) through a variable $R$ isley prism mounted in goggles.

\section{Subjects}

Four male and two female graduate students at the University of New Hampshire served as voluntary subjects.

\section{Procedure}

The subjects sat at arm's length in front of the straight edge. They were informed that the experimenter was interested in finger tracking skill under optically distorted viewing conditions. They were asked to hold their heads still and to run the forefinger of their dominant hands back and forth along a central $48-\mathrm{cm}$ stretch of the straight edge (the viewing lens restricted the field of view to about $48 \mathrm{~cm}$ ). Subjects were told to follow their finger-tracking movements with their eyes. A repetitive auditory click $(0.5 \mathrm{~Hz})$ was presented to the subject before the experimental trials to cue rate of arm movement. The click was not presented during actual experimental trials as it proved distracting and tended to interrupt smooth, continuous tracking motion.

Two minutes of practice under no curve induction viewing conditions allowed the subject to learn the required tracking motion and rate of arm movement. Experimental trials were then run. The experimenter signaled the subject to begin tracking. After five full cycles of lateral finger-tracking motion, the experimenter took a motion picture which included two full cycles of movement.

The motion picture was taken at 14 frames/sec. For measurement purposes, the central $48-\mathrm{cm}$ portion of the straight edge was divided into $104.8-\mathrm{cm}$ intervals, which were clearly demarcated so that they could be included in the photographic record but were not visible to the subject. Since, on the average, the subject traversed the $48 \mathrm{~cm}$ in $1 \mathrm{sec}(1 / 2 \mathrm{cycle})$, the 14 frames $/ \mathrm{sec}$ film speed insured obtaining a pressure record for each of the 10 finger-position intervals.

Subjects viewed the straight edge and their arm movements through the lens first, with a zero curve inducing setting, then a 30 diopter convex-down setting, and finally a 30 diopter convex-up setting. Order effects were not assessed in this experiment. Data for each of the two cycles of movement on a viewing condition trial were averaged prior to analysis.

The experimental design consisted of three variables: direction of curvature (none, convex-down, convex-up), direction of arm movement (toward or away from the body), and finger displacement interval ( 10 intervals from initiation to termination of movement). The factorial combination of these three variables resulted in 60 repeated measures per subject. Even though subjects' arms were always moving back and forth laterally, different arm and shoulder muscle groups would be responsible for movement to the right or the left, depending upon whether the subject was right- or left-handed. Hence. the second design factor classified arm movement as roughly toward or away from the body.

\section{RESULTS}

All subjects reported that the objectively straight edge felt curved in the same direction as the induced optical curvature. The overall mean pressure effects are presented in Figure 2. An analysis of variance of the data revealed three significant first-order interactions: Direction of Curvature by Direction of Arm Movement $[F(2.10)=4.41 . p<.05]$. Direction of Curvature by Finger Displacement Interval $[F(18.90)=9.82 . p<.01]$, and Direction of Arm Movement by Finger Displacement Interval [F(18.45) $=8.07 . \mathrm{p}<.011$.

The interaction most directly related to the experimental hypothesis is that between the direction of curvature and finger displacement interval factors. It can be seen that pressure changes along the straight edge differed depending upon the viewing condition. In general. when the eye receives a convex-down image, finger-tracking pressure is greater at the center of the straight edge. When the eye receives a convex-up image. finger-tracking pressure tends to be greater at the ends of the straight edge. Although the two other interactions do have qualifying effects on finger pressure differences along the straight edge (i.e., whether tracking movement is being initiated vs. terminated or is roughly toward vs. away from the body) and are of interest. for the present purposes the data can be reorganized to provide a more concise description of the major effects. 


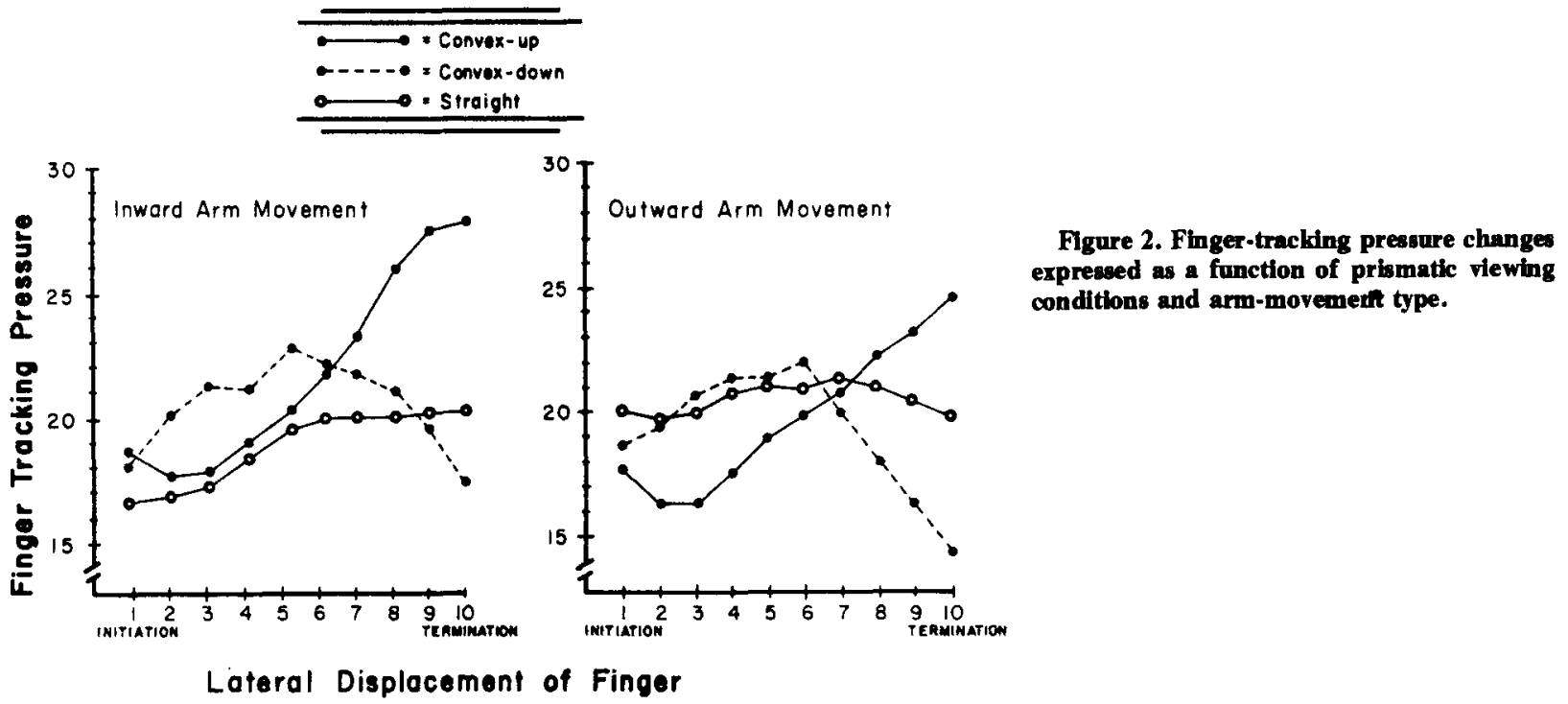

A trend analysis (Winer, 1971) of the six pressure protiles depicted in Figure 2 revealed, among other things, that the quadratic trend components for each viewing condition did not differ significantly across the direction of arm movement factor. Thus the data were first collapsed across that distinction, since the quadratic component of the pressure profiles was of key interest due to the optically created curved visual image. Moreover, since the pressure profiles corresponding to the straight viewing condition were not flat or straight (neither profile, however, had a signiticant quadratic component), the data for the convex-down and convex-up viewing conditions were expressed as deviations by subtracting the straight profile values from the curved profile values. The mean deviations are presented in Figure 3. An analysis of variance of this deviation data revealed no significant main effects of the two design factors. direction of curvature (convex-down vs. convexup) and finger displacement interval (1-10). There was, however, a significant interaction between the factors $[F(9.45)=12.2, p<.001]$. As can be seen, the pressure deviation profiles are inversely related to the visual image curves. It should also be noted that the deviation profiles are not symmetrical. as pressure is disproportionally decreased or increased at the termination of movement.

One final transformation of the data which removed the initiation-termination distinction was performed. Rather than expressing finger displacement as initiation through termination of movement. the 10 intervals can be made to correspond to the spatial positions right and left of the subject's median plane. Deviation data in this form are presented in Figure 4. Interval 1 now corresponds to tracking movement to the subject's left and Interval 10 to tracking movement to the subject's right. An analysis of variance of this data revealed no significant main effects, but the interaction between the two design factors was significant $[F(9.45)=6.3, p<.01]$. A complete trend analysis of the data in this final form indicated the following. The linear component of the Direction of Curvature by Finger Displacement Interval interaction was not significant. The quadratic component of the Direction of Curvature by Finger Displacement Interval interaction was significant $[F(1,5)=8.40, \mathrm{p}<.05]$ and accounted for about $97 \%$ of the variation attributable to the interaction (initial analysis of variance on deviations). The quadratic components of the convex-down and convex-up pressure deviation profiles were signifi-

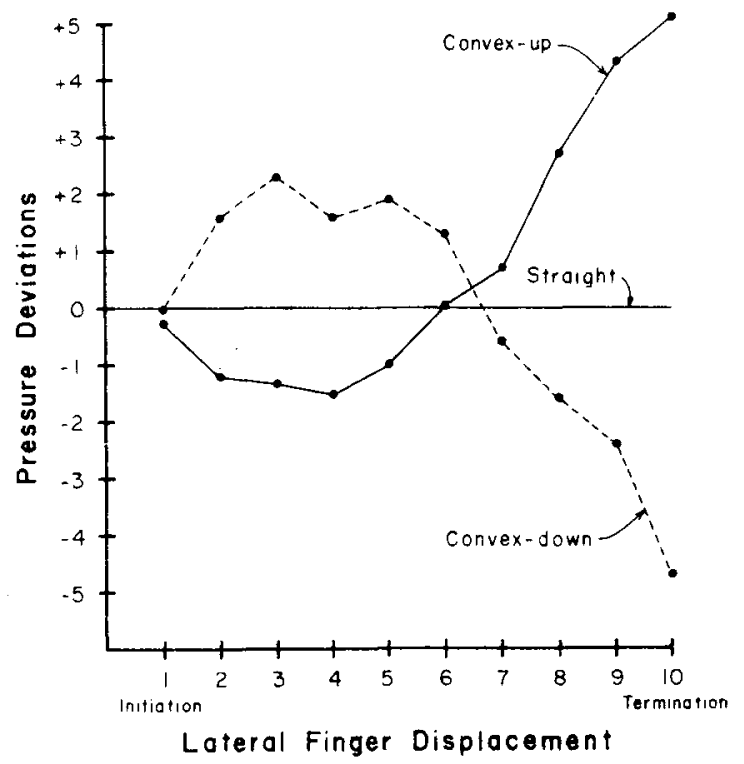

Figure 3. Finger-tracking pressure changes expresced as deviations from the straight viewing condition. The lateral displacement intervals refer to the initiation and termination of movement. 


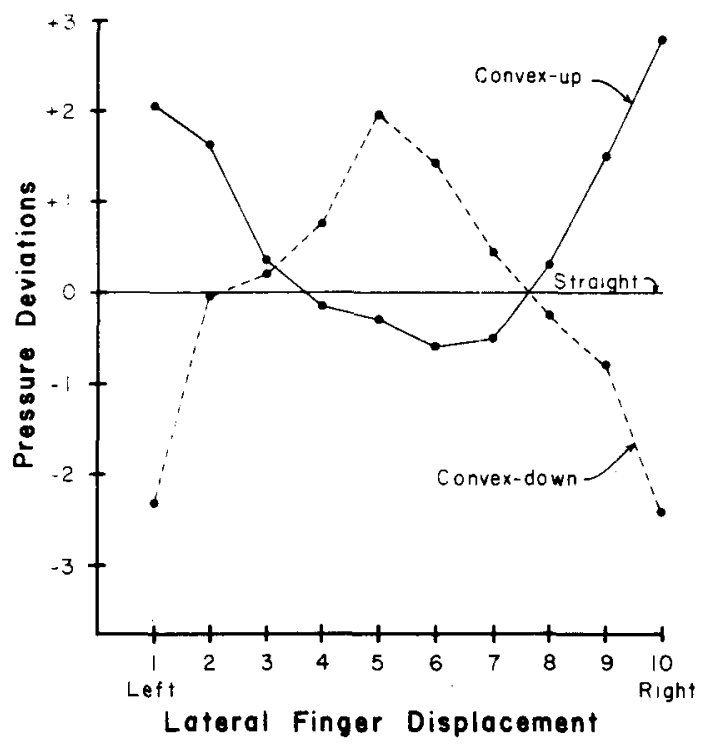

Figure 4. Finger-tracking pressure changes expressed as deviations from the straight viewing condition. The lateral displacement intervals refer to the spatial positions right and left of the subjects' median plane.

cantly different from one another due to their opposite directions $[F(1.45)=55.01, p<.001]$. And finally, the quadratic component of each deviation profile was significantly different from zero $\left[\mathrm{F}_{-} \mathrm{CD}^{(1,90)}=\right.$ 34.32. $\left.\mathrm{p}<.001 ; \mathrm{F}_{-\mathrm{CU}}(1,90)=25.40, \mathrm{p}<.001\right]$.

The upshot of the above analysis is that when pressure changes accompanying finger tracking under straight viewing conditions are removed from the curved-image data, the finger-tracking pressure profiles are inversely related to the visual image curves. It follows that in order to create such pressure profiles the actual tracking movement of the arm must be isomorphic with the visual curves. As mentioned previously, those factors that do influence naturally occurring finger tracking pressure changes such as direction of arm movement and initiation vs. termination of movement need to be assessed but are not of immediate relevance (other than to note their existence) to the present report.

\section{DISCUSSION}

The results indicate that the arm, hand, and finger, in tandem, do what the visual image signals them to do. This finding bears on the nature of the mechanism underlying visual capture. One interpretation, prevalent in the literature, is that vision literally swamps proprioception. That is, a subject feels a straight edge as curved when optically distorted in spite of the fact that his finger is moving in an objectively straight path (Gibson, 1933). The implication of this view is that visual afference is the direct determinant of felt curvature.

The results of the present study would appear to qualify such a position, since it was shown that the arm and hand do not move in a straight path, indicating that proprioceptive afference (active or passive) could result in the experienced curvature. However, in Gibson's experiment, a straight edge which was rigidly fixed in space was tracked. In the present experiment, an edge which was stiffly suspended in space but movable was used in order to index finger pressure. Since "movement" of the edge and finger pressure were confounded, Gibson's position cannot be ruled out. Some control for the effects of the stiffness of the straight edge suspension on felt curvature would clearly be desirable.

Be that as it may, the present results do indicate that when a stiffly suspended straight edge is tracked with the finger under optically induced curvature viewing conditions, curved arm movement results which is correlated with felt curvature. Since the arm is moving in a curved path, three sources of information other than visual afference could cause felt curvature. Passive proprioception (pressure) from the finger, active proprioception from the muscles, joints, and tendons of the arm, or efferent commands to the arm could result in the experience of curvature. The important conclusion of the present study is that passive proprioception does not result in the felt curvature obtained in this experiment. If such information was being monitored, it would result in experienced curvatures exactly opposite in orientation to those which were experienced by subjects and corresponded to the curved visual image.

The contribution of the other sources of information have not yet been determined. The evidence indicates that proprioceptive afference signaling the appropriate curvature is potentially available when subjects track an apparently curved edge. However, in view of the role played by efference in adaptation to prismatic distortion (Festinger et al., 1967; Gyr \& Wiley, 1970), it is entirely plausible that some record of outgoing "instructions" to the arm is responsible for felt curvature.

The results suggest that in the type of visual capture explored here, an intersensory compromise is achieved by the perceptual system not unlike other perceptual constances. Ordinarily, vision and touch are in close, though not exact, correspondence. When the correspondence decreases or a conflict is encountered, the system moves for a resolution which maintains constancy among different sensory information and in experience. In the present context, distortion of information in the visual channel would modify schema common to all senses, with the result that finger-tracking movement would be appropriately curved and congruous with vision. 


\section{REFERENCES}

Festinger, L., Burnham, C. A., Ono, H., \& Bamber, D. Efference and the conscious experience of perception. Journal of Experimental Psychology Monograph, 1967, 74(4, Whole No. 637).

GiBson, J. J. Adaptation, after-effect, and contrast in the perception of curved lines. Journal of Experimental Psychology, $1933,16,1-31$

GYR, J. W., \& WILEY, R. The effects of efference to the arm on visual adaptation to curvature: A replication. Psychonomic Science, 1970, 21, 89-91.

Nielsen, T. I. Volition: A new experimental approach. Scandinavian Journal of Psychology, 1963, 4, 225-230.

Pick, H. L., Warren, D. H., \& Hay, J. C. Sensory conflicts in judgments of spatial direction. Perception \& Psychophysics, $1969,6,203-205$.
Rock, I. The nature of perceptual adaptation. New York: Basic Books, 1966.

Rock, I. An introduction to perception. New York: Macmillan, 1975.

RYAN, T. A. Interrelations of the sensory systems in perception. Psychological Bulletin, 1940, 37, 659-698.

WINER, B. J. Statistical principles in experimental design (2nd ed.). New York: McGraw-Hill, 1971.

(Received for publication June 20, 1975; revision received October 24,1975 .) 